\title{
Reduction in extrapulmonary tuberculosis in context of antiretroviral therapy scale-up in rural South Africa
}

\author{
J. C. HOOGENDOORN ${ }^{1}$, L. RANOTO ${ }^{1}$, N. MUDITAMBI ${ }^{1}$, J. RAILTON ${ }^{1}$, \\ M. MASWANGANYI ${ }^{2}$, H. E. STRUTHERS ${ }^{1,3}$, J. A. MCINTYRE ${ }^{1,4}$ AND \\ R. P. H. PETERS ${ }^{1,5,6 *}$ \\ ${ }^{1}$ Anova Health Institute, Johannesburg, South Africa \\ ${ }^{2}$ Mopani Department of Health, Giyani, South Africa \\ ${ }^{3}$ Department of Medicine, University of Cape Town, Cape Town, South Africa \\ ${ }^{4}$ School of Public Health and Family Medicine, University of Cape Town, Cape Town, South Africa \\ ${ }^{5}$ Department of Medical Microbiology, University of Pretoria, Pretoria, South Africa \\ ${ }^{6}$ Department of Medical Microbiology, CAPRHI School for Public Health and Primary Care, Maastricht \\ University, Maastricht, The Netherlands
}

Received 28 February 2017; Final revision 30 May 2017; Accepted 29 June 2017; first published online 27 July 2017

\section{SUMMARY}

Scale-up of antiretroviral therapy (ART) for human immunodeficiency virus (HIV) infection has reduced the incidence of pulmonary tuberculosis (PTB) in South Africa. Despite the strong association of HIV infection with extrapulmonary tuberculosis (EPTB), the effect of ART on the epidemiology of EPTB remains undocumented. We conducted a retrospective record review of patients initiated on treatment for EPTB in 2009 (ART coverage <5\%) and 2013 (ART coverage $41 \%$ ) at four public hospitals in rural Mopani District, South Africa. Data were obtained from TB registers and patients' clinical records. There was a 13\% decrease in overall number of TB cases, which was similar for cases registered as EPTB ( $n=399$ in 2009 vs. 336 in 2013; $P<0 \cdot 01)$ and for PTB (1031 vs. 896; $P<0 \cdot 01)$. Among EPTB cases, the proportion of miliary TB and disseminated TB decreased significantly (both $P<0 \cdot 01$ ), TB meningitis and TB of bones increased significantly $(P<0 \cdot 01$ and $P=0 \cdot 02$, respectively) and TB pleural effusion and lymphadenopathy remained the same. This study shows a reduction of EPTB cases that is similar to that of PTB in the context of the ART scale-up. The changing profile of EPTB warrants attention of healthcare workers.

Key words: Antiretroviral therapy, extrapulmonary tuberculosis, human immunodeficiency virus, pulmonary tuberculosis, rural South Africa.

\section{INTRODUCTION}

The tuberculosis (TB) epidemic remains a major global health problem despite strong worldwide efforts to control it [1]. In 2012, an estimated 8.6 million people

\footnotetext{
* Author for correspondence: R. P. H. Peters, Anova Health Institute, 12 Sherborne Road, Johannesburg, 2193, South Africa. (Email: peters@anovahealth.co.za)
}

developed TB globally and 1.3 million died from the disease [2]. South Africa has the highest incidence rate per capita with one new case for every 100 people each year [2]. Moreover, South Africa has the highest burden of people coinfected with TB and human immunodeficiency virus (HIV) [2, 3]. The HIV epidemic has been an important driver of the incidence of TB, attributing to the fourfold increase in TB notification in South Africa between 1986 and 2007 [4]. 
In recent years, the antiretroviral therapy (ART) programme for HIV infection has expanded dramatically in South Africa [5], accompanied by a higher CD4 count threshold for treatment initiation [6] and resulting in more HIV-infected patients initiated on ART [7]. Several studies show a reduction in TB incidence among HIV-infected patients in the context of this ART scale-up in South Africa [8-10].

There is evidence for a decrease in incidence of pulmonary tuberculosis (PTB) during ART scale-up in South Africa. Nanoo et al. reports a 9\% decline of PTB between 2008 and 2012, coincided with an increase in ART-coverage [11]. One study reports that the decline in PTB during ART scale-up contributed to an increase in life expectancy among HIV-infected patients [12]. However, contrary to PTB, there is no data on the effect of ART scale-up on the incidence of extrapulmonary TB (EPTB) and the distribution of its different manifestations. There is an important relationship between HIV and EPTB $[13,14]$ : EPTB constitutes $40-68 \%$ of all TB cases among HIV-infected patients, whereas only $15-20 \%$ of all TB cases in immunocompetent patients is EPTB [15-18].

Of important note is that most data on EPTB are from countries outside of sub-Saharan Africa, where the global burden of HIV is concentrated. It is crucial to know more about the epidemiology of EPTB in South Africa. Extrapulmonary manifestation of TB is generally associated with a challenging diagnosis, a considerable diagnostic delay $[19,20]$ and unrecognized and undiagnosed infection [21], all contributing to the documented poor prognosis of EPTB. The aim of this study is to determine the effect of scale-up of the ART programme on the epidemiology of EPTB in a rural population in South Africa, in order to understand the TB epidemic in a better way and to inform TB control efforts and policy design.

\section{METHODS}

\section{Study design and setting}

This is a retrospective record review of patients initiated on treatment for EPTB in 2009 and 2013 at four public hospitals in rural Mopani District, Limpopo Province, South Africa. In this province, the adult HIV prevalence is $12 \%$. We chose the years 2009 and 2013 as these reflect different phases in ART scale-up in South Africa and to allow for TB treatment outcome data to be included in this analysis. In 2009, the ART programme in South Africa was quite small, doctor-driven, and provided at accredited healthcare facilities only. The CD4 count threshold for ART initiation at that time was 200 cells $/ \mathrm{mm}^{3}$; this was changed in 2010 to 350 cells/ $\mathrm{mm}^{3}$ and accompanied by large scale-up of the ART programme through implementation of the nursemanaged ART programme [22].

In our setting, EPTB is almost exclusively diagnosed at hospitals as diagnostic resources and clinical expertise (doctor) are required that is not available at the primary healthcare (PHC) level. We included all seven hospitals (six districts and one regional hospital) in this evaluation. However, the complete required TB register could not be retrieved at three district hospitals and these were excluded from further evaluation.

We obtained ethical approval from the Human Research Committee at the University of Witwatersrand (reference no. M141012).

\section{Data collection}

Data were collected for the months January-October of the years 2009 and 2013 from two sources: the routine TB register and clinical records. The complete TB register could not be retrieved for November and December for all hospitals.

In South Africa, each healthcare facility has a paperbased TB register in which all individuals initiated on $\mathrm{TB}$ treatment are recorded by the TB nurse. We recorded the total number of patients treated for $\mathrm{TB}$ and the number treated for ЕРTB, and for which type, as defined by using the International Statistical Classification of Diseases and Related Health Problems 10th Revision (ICD-10) [23]. Other information obtained from this register includes data on age, sex, history of TB treatment, HIV co-infection, ART usage at treatment initiation, CD4 count at treatment initiation and treatment outcome.

In addition to the information from the TB registers, clinical records of patients treated for EPTB were retrieved and reviewed by JCH, LR and NM using a standardized data collection sheet. We collected additional information on investigations, diagnosis, treatment and comorbidity (e.g. HIV). We used this additional data to validate the data from the TB register and to determine evidence for the given diagnosis.

\section{Definitions}

All ICD-10 codes recorded in the TB register were classified as EPTB except for A15.0 (TB of lung, 
confirmed by sputum microscopy), A16.2 (TB of lung, without mention of bacteriological of histological confirmation) and A16.7 (primary respiratory TB without mention of bacteriological or histological confirmation), which together conferred the group of PTB. For each retrieved clinical record, two investigators (JCH, RPHP) determined the level of evidence available to support the diagnosis written in the clinical record and classified this as 'good evidence', 'limited evidence and 'no evidence'. In case of difference between the investigators, a third investigator (LR) decided. We used the National Tuberculosis Guideline 2014 [24] to determine the level of evidence (Supplementary Table S1). In this context, the comment 'miliary TB picture on X-ray' was defined as miliary TB, whereas 'disseminated TB' was defined as EPTB at multiple extrapulmonary sites.

\section{Data analysis}

All data were double-entered in Epi Info Version 3.5.4. (Centers for Disease Control and Prevention, Atlanta, USA) and analysed using IBM SPSS Statistics Version 22.0 (IBM Corporation, New York, USA). Routine descriptive statistics of the study population are provided using total number with proportion (\%), median (range) and mean [standard deviation (s.D.)]. The number of patients treated for EPTB and the distribution of EPTB forms was compared between 2009 and 2013 using the Z-test for proportions; this was done both for data retrieved from the TB registers and the patients' clinical records. The 95\% confidence intervals (CI) were calculated using the method described by Wilson. To determine the incidence of EPTB at population level, the total population was calculated using available census data for individuals residing in the catchment area hospitals included in this evaluation $(997650$ individuals in 2011) [25]. A $P$-value of $<0.05$ was considered significant.

\section{RESULTS}

\section{TB cases over time in the district}

The total number of TB cases recorded in the TB register decreased by $13 \%(95 \%$ CI $11-15 \%)$ from 1432 patients in 2009 to 1239 patients in 2013 (Fig. 1). At the same time, the estimated mid-year ART-coverage amongst all HIV-infected patients in Mopani District increased from 5\% (5489 patients on ART) in 2009 to

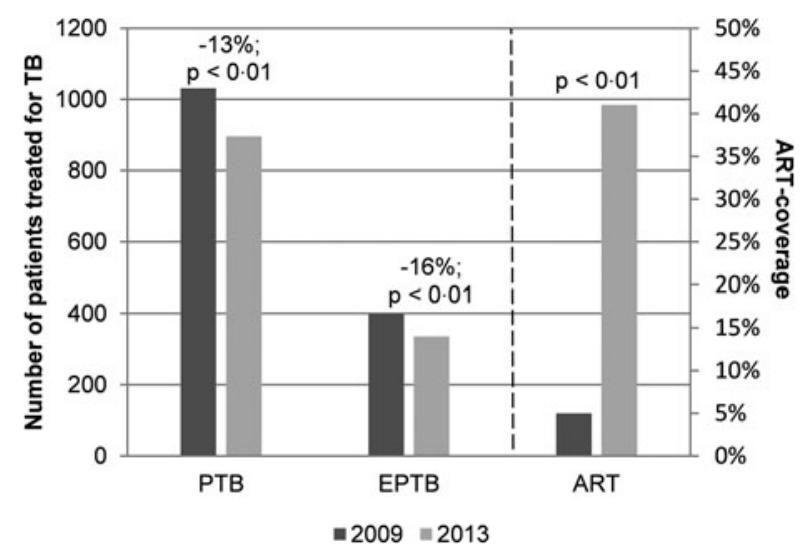

Fig. 1. Number of patients treated for extrapulmonary and pulmonary TB as per hospital TB registers in the context of ART-coverage in a rural district in 2009 and 2013. TB, tuberculosis; EPTB, extrapulmonary tuberculosis; PTB, pulmonary tuberculosis; ART, antiretroviral therapy.

41\% (44 015 patients on ART) in $2013(P<0 \cdot 01)$. The reduction was observed for both EPTB (399 vs. 336 cases; $P<0 \cdot 01)$ and PTB (1031 vs. 896 cases; $P<0 \cdot 01)$. The proportion of cases classified as EPTB remained the same between both years ( $28 \% v s .27 \% ; P=0.68)$ and the reduction in cases was in the same range for EPTB $[16 \%(95 \%$ CI $13-20 \%)]$ and PTB $[13 \%$ (95\% CI $11-15 \%) ; P=0 \cdot 59]$.

\section{Demographic characteristics of patients treated for EPTB over time}

The decrease in EPTB cases was based on a $21 \%$ decrease among adult individuals (from 373 in 2009 to 295 in 2013) (Table 1). However, although the absolute number is small, the number of children treated for EPTB increased from 26 in 2009 to 41 in 2013 $(P<0 \cdot 01)$. Amongst these children the proportion HIV-uninfected children treated for EPTB increased significantly from 8 in 2009 to 27 children in 2013 ( $31 \%$ vs. $66 \% ; P<0.01)$; this was not due to a specific type of ЕРTB. There was no difference in gender distribution over time. The proportion of individuals with unknown HIV status decreased over time, but among those with documented HIV status, the proportion of individuals with EPTB that had a positive HIV status decreased $(P<0 \cdot 01)$. The ART-coverage among HIV-infected patients with EPTB increased from $25 \%$ to $57 \%(P<0 \cdot 01)$ between both years. In $2009,18 \%$ of all patients classified as EPTB were HIV-uninfected, increasing to $30 \%$ in 2013 $(P<0 \cdot 01)$; this was not related to gender. The median 
Table 1. Characteristics of patients treated for extrapulmonary tuberculosis

\begin{tabular}{|c|c|c|c|}
\hline & $\begin{array}{l}2009 \\
(n=399)\end{array}$ & $\begin{array}{l}2013 \\
(n=336)\end{array}$ & $P$-value \\
\hline \multicolumn{4}{|l|}{ Age $^{\mathrm{a}}$} \\
\hline$\leqslant 18$ years & $26(7)$ & $41(12)$ & \multirow[t]{2}{*}{$P<0.01$} \\
\hline$>18$ years & $373(93)$ & $295(88)$ & \\
\hline \multicolumn{4}{|l|}{ Gender $^{\mathrm{a}}$} \\
\hline Male & $189(47)$ & $150(45)$ & \multirow[t]{2}{*}{$P=0 \cdot 48^{\mathrm{b}}$} \\
\hline Female & $210(53)$ & $185(55)$ & \\
\hline \multicolumn{4}{|l|}{ HIV-infection ${ }^{\mathrm{a}}$} \\
\hline HIV-infected & $267(82)$ & $224(70)$ & $P<0 \cdot 01^{\mathrm{c}}$ \\
\hline HIV-uninfected & $57(18)$ & $94(30)$ & \multirow{2}{*}{$P<0.01^{\mathrm{d}}$} \\
\hline Unknown & 75 & 18 & \\
\hline \multicolumn{4}{|c|}{ HIV-infected individuals on $\mathrm{ART}^{\mathrm{a}}$} \\
\hline Yes & $63(25)$ & $123(57)$ & \multirow[t]{2}{*}{$P<0.01^{\mathrm{e}}$} \\
\hline No & $192(75)$ & $94(43)$ & \\
\hline \multicolumn{4}{|c|}{ Median CD4 of HIV-infected patients at diagnosis of EPTB } \\
\hline On ART & $117(16-362)$ & $103(4-725)$ & \\
\hline Not on ART & $127(3-845)$ & $123(1-1149)$ & \\
\hline
\end{tabular}

HIV, human immunodeficiency virus; ART, antiretroviral therapy.

${ }^{a}$ Data are shown as number (\%).

${ }^{\mathrm{b}} P$-value is given for male $v s$. female.

${ }^{\mathrm{c}} P$-value is given for HIV-infected $v s$. HIV-uninfected.

${ }^{\mathrm{d}} P$-value is given for known HIV-status $v s$. unknown HIV-status.

${ }^{\mathrm{e}} P$-value is given for Yes $v s$. No.

${ }^{\mathrm{f}}$ Data are shown as median (minimum-maximum) in cells $/ \mathrm{mm}^{3}$.

CD4-count was slightly lower amongst HIV-infected patients on ART compared with those not on ART and remained the same between both years. For both years $80 \%$ of the treatment outcome was missing, mainly due to patients moving out to primary health clinics for follow-up.

\section{Distribution of types of EPTB}

TB pleural effusion is the most common type of EPTB ( $53 \%$ in 2009 vs. $56 \%$ of all cases in 2013), followed by miliary TB (30\% respectively 14\%) (Fig. 2a). There was no significant change of the proportion of individuals treated for pleural effusion over time, but the number of cases of miliary TB decreased significantly from 119 to $49(P<0 \cdot 01)$. The proportion of individuals treated for miliary TB that had a positive HIV status remained the same $(89 \%$ vs. $80 \%, P=0 \cdot 15$, Table 2). The ART-coverage increased from $26 \%$ to $62 \%(P<0 \cdot 01)$. The median CD4-count remained very low for both years and both HIV-infected people on ART and not on ART amongst these patients with miliary TB.

The other types of TB were recorded in relatively small numbers. However, in contrast to the reduction in miliary $\mathrm{TB}$, the proportion of patients with $\mathrm{TB}$ meningitis increased significantly from 10 to 33 patients between 2009 and $2013 \quad(P<0 \cdot 01)$; the increase in TB meningitis was not associated with HIV status $(P=0 \cdot 50$, Table 2$)$. TB of bones and joints also increased significantly between both years from 5 to 13 patients $(P=0 \cdot 02)$. No significant changes were observed in the small number of cases of TB lymphadenitis and TB of unspecified organs.

\section{Diagnosis in TB registers vs. clinical records}

We retrieved 253 clinical records of 399 patients $(63 \%)$ classified as EPTB in the TB register in 2009 and 223 clinical records of 336 patients $(66 \%)$ classified as EPTB in the register in $2013(P=0 \cdot 40)$ (Fig. 3).

Information with regards to diagnosis was missing in four clinical records in 2009 and eight in 2013. In $2009,88 \%$ of the patients had the same EPTB diagnosis recorded in register and patient's clinical record, compared to $69 \%$ in $2013(P<0 \cdot 01)$. Amongst the 30 patients with a discordant diagnosis between register and patient's clinical record 16 were diagnosed PTB in 2009, compared with 39 of the 64 patients in $2013(P=0 \cdot 48)$. The most common ICD-10 code of these patients in the TB register was TB pleural effusion, followed by miliary TB. The remaining patients with different diagnosis had a diagnosis of EPTB in both clinical record and register, but at a different site.

The trends in population characteristics (e.g. gender, age and HIV status) were similar as recorded from the registers and the patients' clinical records, including a significant increase in children treated for EPTB and a significant increase in HIV-uninfected patients treated for EPTB. This supports validity of data captured from both resources. Based on the diagnosis recorded in the clinical records, the same trends in type of EPTB over time were generally observed as reported from the TB register (Fig. 2b). The only difference observed was for TB lymphadenitis with an increase from $7 \%$ to $17 \% \quad(P<0.01)$ in cases according to the information retrieved from the clinical records. However, we did not manage to retrieve the clinical record from 10 of the 23 patients $(43 \%)$ classified as TB lymphadenitis in 2009 according to the register compared with 5 of the 28 patients $(18 \%)$ in $2013(P=0 \cdot 05)$. 

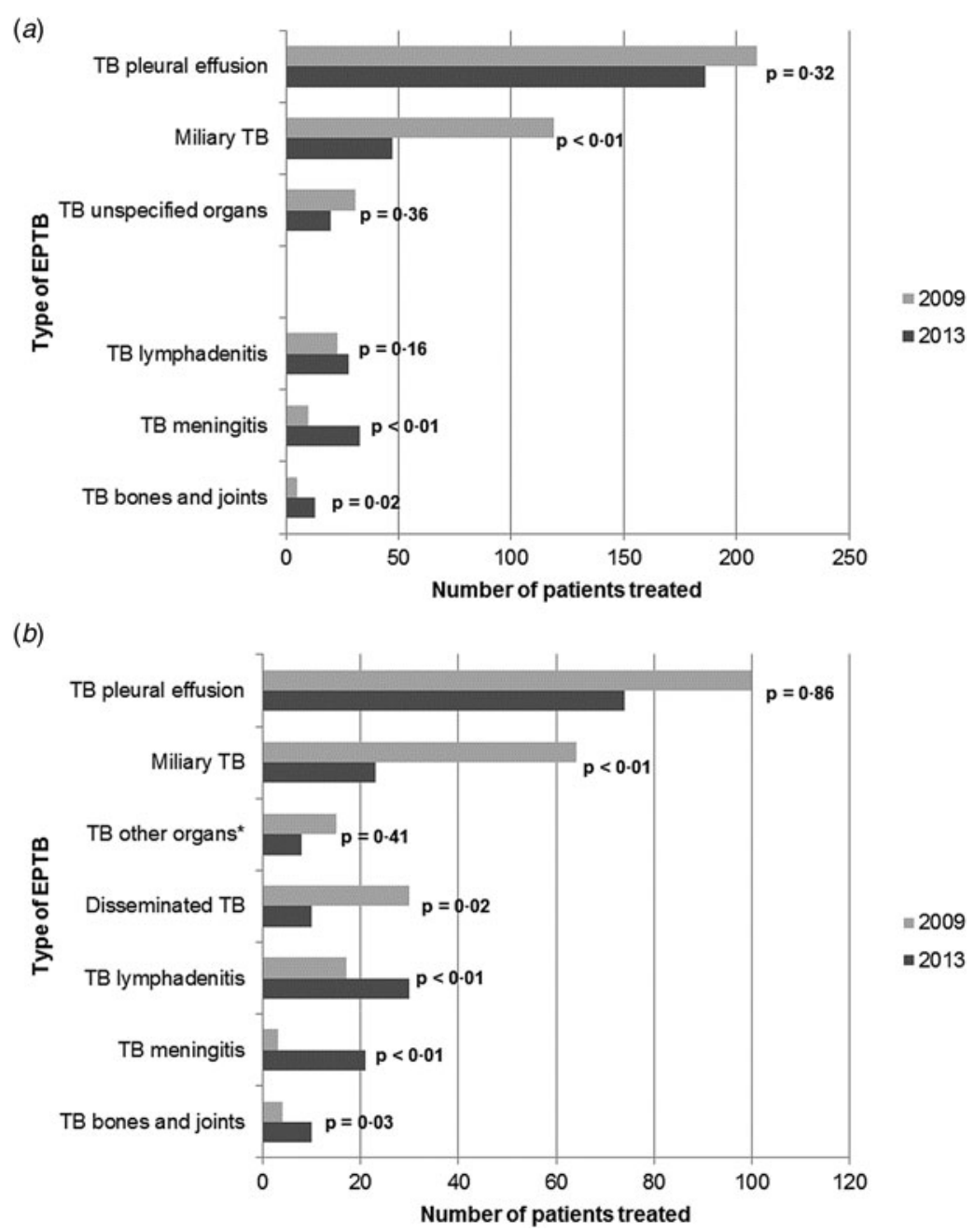

Fig. 2. Distribution of types of extrapulmonary tuberculosis recorded at four hospitals in Mopani District between 2009 and 2013. (a) Data from hospital TB register. (b) Data from clinical records. TB, tuberculosis; EPTB, extrapulmonary tuberculosis. $P$-value given as compared with total EPTB cases. *Based on the diagnosis in clinical records these include cases of TB abdomen (eight cases in 2009 vs. six cases in 2013), TB pericarditis (five cases vs. two cases, respectively), TB mastitis (one case $v s .0$ cases, respectively) and TB artery (one case $v s .0$ cases, respectively).

As per clinical records there was a significant decrease in disseminated TB $(13 \%$ in 2009 vs. $6 \%$ in 2013, $P=0 \cdot 02)$. The proportion of HIV-infected patients remained the same amongst this subpopulation $(P=0.76)$, while the ART-coverage increased significantly $(P<0 \cdot 01)$.

\section{Evidence for given diagnosis}

In 136 of the 233 retrieved clinical records in $2009(59 \%)$ and 95 of the 176 clinical records in 2013 (55\%), there was good evidence available to support the diagnosis. There was limited evidence in $28 \%$ vs. $32 \%$ of the clinical records respectively and no evidence supporting the diagnosis in $13 \%$ vs. $13 \%$ of the clinical records respectively. This did not differ significantly between both years $(P=0 \cdot 61)$. The evidence and investigations for miliary TB and TB of bone and spine were documented very well with $95 \%$ vs. $71 \%$ good evidence for the diagnosis, respectively. TB lymphadenitis was recorded the worst with no evidence for the diagnosis in the retrieved clinical record in $41 \%$ of all cases of TB lymphadenitis. The evidence for TB meningitis could not be compared between both years due to small numbers in 2009 (three cases only), although $75 \%$ of the cases with TB meningitis in 2013 had good or limited evidence for the diagnosis (Table 3).

\section{DISCUSSION}

This is the first study that demonstrates a significant decrease in cases of EPTB in the context of ART 
Table 2. Types of extrapulmonary tuberculosis in 2009 and 2013 according to the ICD-10-codes in the TB register stratified for HIV infection

\begin{tabular}{|c|c|c|c|}
\hline & $2009(n=399)$ & $2013(n=336)$ & $P$-value \\
\hline \multicolumn{4}{|l|}{ TB pleural effusion ${ }^{a}$} \\
\hline HIV-infected & $128(78)$ & $129(72)$ & \multirow[t]{2}{*}{$P=0.21^{\mathrm{t}}$} \\
\hline HIV-uninfected & $37(22)$ & $51(28)$ & \\
\hline Unknown & 44 & 6 & $P<0.01^{\mathrm{c}}$ \\
\hline \multicolumn{4}{|l|}{ Miliary $\mathrm{TB}^{\mathrm{a}}$} \\
\hline HIV-infected & $89(89)$ & $36(80)$ & \multirow{2}{*}{$P=0 \cdot 15^{\mathrm{b}}$} \\
\hline HIV-uninfected & $11(11)$ & $9(20)$ & \\
\hline Unknown & 19 & 2 & $P=0.04^{\mathrm{c}}$ \\
\hline \multicolumn{4}{|l|}{ TB lymphadenitis ${ }^{\mathrm{a}}$} \\
\hline HIV-infected & $17(89)$ & $14(50)$ & \multirow[t]{2}{*}{$P=0 \cdot 01^{\mathrm{b}}$} \\
\hline HIV-uninfected & $2(11)$ & $14(50)$ & \\
\hline Unknown & 4 & 0 & $P=0.02^{\mathrm{c}}$ \\
\hline \multicolumn{4}{|l|}{ TB meningitis ${ }^{\mathrm{a}}$} \\
\hline HIV-infected & $6(67)$ & $21(78)$ & \multirow{2}{*}{$P=0 \cdot 50^{\mathrm{b}}$} \\
\hline HIV-uninfected & $3(33)$ & $6(22)$ & \\
\hline Unknown & 1 & 6 & $P=0.54^{\mathrm{C}}$ \\
\hline \multicolumn{4}{|l|}{ TB bones and joints ${ }^{\mathrm{a}}$} \\
\hline HIV-infected & $3(100)$ & $4(40)$ & \multirow{2}{*}{$P=0.07^{\mathrm{t}}$} \\
\hline HIV-uninfected & 0 & $6(60)$ & \\
\hline Unknown & 2 & 3 & $P=0.47^{\circ}$ \\
\hline \multicolumn{4}{|c|}{ TB of unspecified organs $\mathrm{s}^{\mathrm{a}}$} \\
\hline HIV-infected & $24(89)$ & $14(64)$ & \multirow[t]{2}{*}{$P=0.03^{\mathrm{t}}$} \\
\hline HIV-uninfected & $3(11)$ & $8(36)$ & \\
\hline Unknown & 4 & 1 & $P=0.28^{\mathrm{c}}$ \\
\hline \multicolumn{4}{|l|}{ Missing diagnosis ${ }^{\mathrm{a}}$} \\
\hline HIV-infected & 0 & $6(100)$ & \multirow[t]{2}{*}{$P<0.01^{\mathrm{b}}$} \\
\hline HIV-uninfected & $1(100)$ & 0 & \\
\hline Unknown & 1 & 0 & $P<0.01^{\mathrm{c}}$ \\
\hline
\end{tabular}

TB, tuberculosis; HIV, human immunodeficiency virus.

${ }^{\mathrm{a}}$ Data are shown as number (\%).

${ }^{\mathrm{b}} P$-value is given for HIV-infected $v s$. HIV-uninfected.

${ }^{\mathrm{c}} P$-value is given for known HIV-status $v$ s. unknown HIV-status.

scale-up in Africa. We show that the decrease in EPTB in our setting is similar to that of PTB and that the epidemiological profile of EPTB has changed. We used routinely available data of the TB register in this analysis and confirmed the observation by review of patients' clinical records to assess data quality issues and concerns regarding the clinical diagnostic evidence of diagnosis.

The HIV epidemic has been a major driver of the TB epidemic in Africa [4]. In that context, provision of ART is an important intervention to reduce the burden of TB. Several studies suggest that the TB control programme might benefit from an expanded access to ART in HIV-infected patients [9, 26]. We observed a 13\% decline in TB cases between 2009 and 2013 in the context of the ART scale-up in a rural South African district. This is in line with other studies: a study from Malawi reported a decrease of $33 \%$ of TB cases five years after introduction of ART [27] and in Cape Town the TB notification rate declined by $16 \%$ [10]. Furthermore, Nanoo et al. reported a decline of microbiologically confirmed PTB of 9\% between 2008 and 2012 in South Africa in the context of the ART scale-up [11], similar to the $13 \%$ decrease in PTB we observed.

We provide the first report that shows a similar decline in cases of EPTB as for PTB in our setting. Despite that this is a promising observation we hoped to find a decline in EPTB that would be larger than for PTB. HIV has a stronger relationship with EPTB than PTB [14, 28]. Gomer et al. report an Odds Ratio (OR) $2 \cdot 15$ (95\% CI $2 \cdot 09-2 \cdot 21)$ of HIV infection amongst EPTB comparing with PTB. A possible explanation of our findings is an overestimation of the decline in PTB patients, because we only analyzed hospital data and did not include data from 


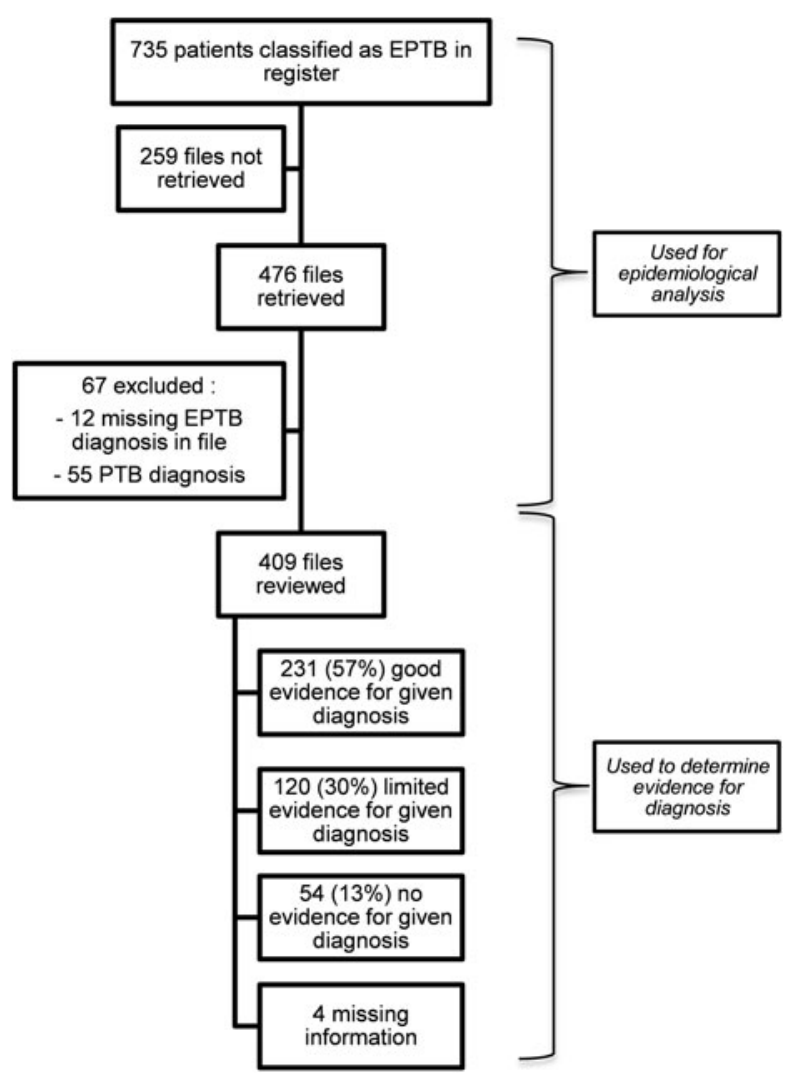

Fig. 3. Flow chart of review of clinical records. EPTB, extrapulmonary tuberculosis; PTB, pulmonary tuberculosis.

PHC settings. Generally, patients diagnosed with PTB at the hospital have more severe illness than the uncomplicated cases at PHC facilities. A shift towards less severe TB disease as result of ART could have let to more individuals being diagnosed with PTB outside the hospital. The introduction of GeneXpert in 2010 as first-line diagnostic for TB might have contributed to more diagnosis of PTB in the PHC as well [24, 29].

We observed an increase in EPTB amongst children. Although this is surprising, especially amongst HIV-uninfected children, we were not able to relate this increase to any specific hospital or the pattern in the distribution of EPTB. We do not have a clear explanation for this, but increased diagnostic awareness among local doctors may not be ruled out. In our study, the median CD4-count remained the same between both years. This illustrates the strong association of EPTB with low CD4 count in HIV-infected individuals. Although population risk for EPTB was reduced following ART scale-up (as reflected in decreased number of EPTB cases), HIV-infected patients are still at risk of getting EPTB when their CD4 counts are low (ART not started, non-compliance to ART, on ART for a short time) [21].

The most common type of EPTB was TB pleural effusion followed by miliary TB. TB lymphadenitis was only diagnosed in $6 \%$ of the cases in 2009 and $9 \%$ of the cases in 2013. Moreover, in patients treated for TB lymphadenitis there is a low frequency of good documentation supporting this diagnosis in the patient records. Other studies report TB pleural effusion [26, 28, 30, 31] or TB lymphadenitis $[15,32]$ as the most common type of EPTB. This suggests that there is under recording. The under diagnosis of TB lymphadenitis in our region warrants attention.

The absolute number of patients with TB pleural effusion decreased, but it still is the site of EPTB for half of the patients. There are several reasons why there may not be a decrease in the proportion of patients with pleural effusion. The definitive diagnosis is difficult to achieve [33], especially because it requires invasive investigations and GeneXpert has a poor sensitivity in diagnosing TB pleural effusion $[34,35]$. This is also illustrated in our review of clinical records where only $40 \%$ had good evidence for the diagnosis. Besides this, pleural effusion can be reactive to PTB, where it should be classified as PTB instead of EPTB. Finally, the possibility of misdiagnosis of pleural effusion of other aetiology as EPTB (e.g. heart failure and malignancy) cannot be excluded, especially in settings with limited resources.

The HIV epidemic has been associated with a strong increase in miliary and disseminated TB, especially among individuals with severe immunosuppression (CD4 $<200$ cells/ $\mu$ l) [36, 37]. HIV-infection is a strong predictor for blood stream infection (BSI) with Mycobacterium tuberculosis [38]. Our data show a significant reduction in both miliary TB and disseminated TB associated with ART scale-up. This suggests that ART scale-up is decreasing the risk of BSI in HIV-infected patients, which has been reported before [39].

Although we describe an encouraging decline in EPTB in general, and in miliary TB and disseminated TB in particular, we observed a significant increase in the small number of TB of bones and joints and cases of TB meningitis. One study reports a rising incidence of TB of bones and joints; none of the patients was HIV-infected [40]. This may implicate that TB of bones and joints is not related to HIV as strongly as most other types of EPTB and probably other risk factors are more important. HIV-infected patients are at higher risk for $\mathrm{TB}$ meningitis compared with HIV-uninfected patients [41], which led to our 
Table 3. Evidence for given diagnosis in patient's clinical record stratified for type of extrapulmonary tuberculosis

\begin{tabular}{llll}
\hline \hline & $\begin{array}{l}\text { Good evidence for } \\
\text { given diagnosis }\end{array}$ & $\begin{array}{l}\text { Limited evidence for } \\
\text { given diagnosis }\end{array}$ & $\begin{array}{l}\text { No evidence for } \\
\text { given diagnosis }\end{array}$ \\
\hline Miliary TB & $83(95)$ & $2(2)$ & $2(2)$ \\
TB meningitis & $9(41)$ & $8(36)$ & $5(24)$ \\
TB pleural effusion & $69(40)$ & $87(50)$ & $17(10)$ \\
TB bone and spine & $10(71)$ & $4(29)$ & 0 \\
TB lymphadenitis & $17(37)$ & $10(23)$ & $19(41)$ \\
Disseminated TB & $31(78)$ & $3(8)$ & $6(15)$ \\
TB of other organs & $12(52)$ & $6(26)$ & $5(22)$ \\
\hline \hline
\end{tabular}

TB, tuberculosis.

Data are shown as number (\%).

${ }^{\text {a }}$ Including 14 cases of TB abdomen, seven cases of TB pericarditis, one case of TB mastitis and one case of TB artery.

hypothesis TB meningitis would decline in the context of the ART scale-up. However, our data show an increase, which is similar for all four hospitals, while the evidence for TB meningitis in the patient's clinical record remained the same between both years. This trend needs attention and should be investigated in further detail, as TB meningitis is a predictor of mortality amongst HIV-infected patients with EPTB [42].

This study has several limitations. First, we performed a retrospective record review using data from the hospital TB registers. Due to the general lack of resources and skills to conduct a prospective study, we conducted a retrospective analysis of routinely available data. Data quality issues are always a concern when using routine programme data, but by analysing both the TB registers as well as the clinical records we attempted to minimize this effect in our interpretation. TB is a notifiable disease in South Africa and everyone initiated on TB treatment has to be recorded, resulting in a little recording bias when analysing the data from the TB register. However, poor record keeping in files is a well-known phenomenon in African healthcare setting; hence, it is not surprising that recording of evidence of diagnosis is not optimal. These diagnostic issues do not mean that tests were not performed or that the diagnosis is incorrect. It reflects both the quality of record keeping as well as the quality of clinical assessment and diagnosis. Despite some discordance between TB register and clinical records, the trends were similar, providing a good reflection of what is happening over time in our setting. Besides this, the level of ART adherence was not assessed and we cannot exclude a possible influence of this on the epidemiology of EPTB. We also do not have data on the time elapsed between ART initiation and EPTB, so we could not determine the role of immune reconstitution in the epidemiology. Furthermore, we could not retrieve and provide any accurate information about prognosis and follow-up. We choose 2013 as year after the start of the ART scale-up in order to get data about treatment outcome and follow-up. Unfortunately data about follow-up was missing in most of the patients, mainly due to referral back to the primary health care. This is a missed opportunity as ART has been associated with a reduced mortality rate among PTB patients and it would have been important to assess this association in the context of EPTB as well [43-45]. Despite these limitations, this is the first study on this topic, providing insight in the epidemiology of EPTB in the context of the ART scale-up. Prospective data are needed to confirm the observed trends.

Despite the documented reduction in EPTB associated with strong scale-up of ART services in our rural district, a considerable burden of disease remains. Prevention of infection, early detection of $\mathrm{TB}$, accurate diagnosis and treatment, and active screening, especially amongst HIV-infected patients, are necessary to control the TB epidemic [44, 45]. In addition, more awareness is required for the documentation of EPTB data. There should also be awareness for the diagnosis and clinical management of EPTB, especially for the types of ЕРTB where laboratory testing is needed (e.g. TB lymphadenitis and TB pleural effusion).

In conclusion, we provide the first data that show an encouraging decrease in EPTB between 2009 and 2013, similar to PTB, in the context of the ART scale-up in Africa. Despite this reduction a considerable epidemic remains, resulting in an ongoing broad public health implication of TB. Besides this, the epidemiology of EPTB is changing, which shows 
the need for educated healthcare workers and expansion of the TB programme in rural South Africa.

\section{SUPPLEMENTARY MATERIAL}

The supplementary material for this article can be found at https://doi.org/10.1017/S095026881700156X.

\section{ACKNOWLEDGEMENTS}

Anova Health Institute receives a grant from the US President's Emergency Plan for AIDS Relief (PEPFAR) program via the US Agency for International Development under Cooperative Agreement No. AID-674-A-12-00015. The views expressed in this report do not necessarily reflect those of PEPFAR or USAID. The funders had no role in study design, data collection and analysis, decision to publish or preparation of the manuscript. We thank the Mopani Department of Health for their support of this study.

\section{DECLARATION OF INTEREST}

The authors declare no conflict of interest.

\section{REFERENCES}

1. World Health Organization. Global Tuberculosis Report 2013 Supplement: Countdown to 2015. Geneva, Switzerland: World Health Organization, 2013.

2. World Health Organization. Global Tuberculosis Report 2013. Geneva, Switzerland: World Health Organization, 2013.

3. Corbett EL, et al. The growing burden of tuberculosis: global trends and interactions with the HIV epidemic. Archives of Internal Medicine 2003; 163: 1009-1021. doi: 10.1001/archinte.163.9.1009.

4. Abdool Karim SS, et al. HIV infection and tuberculosis in South Africa: an urgent need to escalate the public health response. Lancet 2009; 374: 921-933. doi: 10.1016/ S0140-6736(09)60916-8.

5. Klausner JD, et al. Scale-up and continuation of antiretroviral therapy in South African programs, 2005-2009. Journal of the Acquired Immune Deficiency Syndrome 2011; 56: 292-295. doi: 10.1097/QAI.0b013e3182067d99.

6. World Health Organization. Rapid Advice: Antiretroviral Therapy for HIV Infection in Adults and Adolescents. Geneva, Switzerland: World Health Organization, 2009.

7. Plazy M, et al. Change of treatment guidelines and evolution of ART initiation in rural South Africa: data of a large HIV care and treatment programme. BMC Infectious Diseases 2015; 15: 452. doi: 10.1186/ s12879-015-1207-2.
8. Bachmann MO, Timmerman V, Fairall LR. Effect of antiretroviral treatment on the risk of tuberculosis during South Africa's programme expansion. AIDS 2015; 29: 2261-2268. doi: 10.1097/QAD.0000000000000806.

9. Badri M, Wilson D, Wood R. Effect of highly active antiretroviral therapy on incidence of tuberculosis in South Africa: a cohort study. Lancet 2002; 359: 2059 2064. doi: 10.1016/S0140-6736(02)08904-3.

10. Hermans $\mathbf{S}$, et al. Temporal trends in TB notification rates during ART scale-up in Cape Town: an ecological analysis. Journal of the International AIDS Society 2015; 18: 20240. doi: 10.7448/IAS.18.1.20240.

11. Nanoo A, et al. Nationwide and regional incidence of microbiologically confirmed pulmonary tuberculosis in South Africa, 2004-12: a time series analysis. Lancet Infectious Diseases 2015; 15: 1066-1076. doi: 10.1016/ S1473-3099(15)00147-4.

12. Reniers G, et al. Trends in the burden of HIV mortality after roll-out of antiretroviral therapy in KwaZuluNatal, South Africa: an observational community cohort study. Lancet HIV 2017; 4: e113-e121. doi: 10.1016/ S2352-3018(16)30225-9.

13. Bates M, et al. Burden of tuberculosis at post mortem in inpatients at a tertiary referral centre in sub-Saharan Africa: a prospective descriptive autopsy study. Lancet Infectious Diseases 2015; 15: 544-551. doi: 10.1016/ S1473-3099(15)70058-7.

14. Naing $\mathbf{C}$, et al. Meta-analysis: the association between HIV infection and extrapulmonary tuberculosis. Lung 2013; 191: 27-34. doi: 10.1007/s00408-012-9440-6.

15. Sharma SK, Mohan A. Extrapulmonary tuberculosis. Indian Journal of Medical Research 2004; 120: 316-353.

16. Ghiya R, et al. Clinico-epidemiological profile of HIV/ $\mathrm{TB}$ coinfected patients in Vadodara, Gujurat. Indian Journal of Sexually Transmitted Diseases 2009; 30: 10 15. doi: 10.4103/0253-7184.55472.

17. Kingkaew N, et al. HIV-associated extrapulmonary tuberculosis in Thailand: epidemiology and risk factors for death. International Journal of Infectious Diseases 2009; 13: 722-729. doi: 10.1016/j.ijid.2008.11.013.

18. Wilkinson D, Moore DA. HIV-related tuberculosis in South Africa - clinical features and outcome. South African Medical Journal 1996; 86: 61-67.

19. Lee JY. Diagnosis and treatment of extrapulmonary tuberculosis. Tuberculosis and Respiratory Disease 2015; 78; 47-55. doi: 10.4046/trd.2015.78.2.47.

20. Diendéré EA, et al. Clinical outcomes and mortality associated factors in patients infected with HIV receiving a presumptive anti-tuberculosis treatment in a tertiary level hospital in Burkina Faso. AIDS Care 2015; 27: 1250-1254. doi: 10.1080/09540121. 2015.1050982.

21. Leeds IL, et al. Site of extrapulmonary tuberculosis is associated with HIV infection. Clinical Infectious Diseases 2012; 55: 75-81. doi: 10.1093/cid/cis303.

22. Department of Health South Africa. Clinical Guidelines for the Management of HIV and AIDS in Adults and Adolescents. Department of Health, 2010.

23. World Health Organization. International statistical classification of diseases and related health problems 
10th revision: version for 2008 (http://apps.who.int/classifications/icd10/browse/2008/en\#/A15-A19). Accessed 24 January 2017.

24. Department of Health South Africa. National Tuberculosis Management Guidelines 2014. Pretoria, South Africa: Department of Health, 2014.

25. Statistics South Africa (Stats SA). (http://www.statssa. gov.za/). Accessed 24 January 2017.

26. Ade $\mathbf{S}$, et al. National profile and treatment outcomes of patients with extrapulmonary tuberculosis in Bénin. PLOS ONE 2014; 9: e95603. doi: 10.1371/journal. pone.0095603.

27. Zachariah R, et al. Reduced tuberculosis case notification associated with scaling up antiretroviral treatment in rural Malawi. International Journal of Tuberculosis and Lung Disease 2011; 15: 933-937. doi: 10.5588/ijtld.10.0666.

28. Gomes T, et al. Epidemiology of extrapulmonary tuberculosis in Brazil: a hierarchical model. BMC Infectious Diseases 2014; 14: 9. doi: 10.1186/1471-2334-14-9.

29. Meyer-Rath G, et al. The impact and cost of scaling up GeneXpert MTB/RIF in South Africa. PLoS ONE 2012; 7: e36966. doi: 10.1371/journal.pone.0036966.

30. García-Rodríguez JF, et al. Extrapulmonary tuberculosis: epidemiology and risk factors. Enfermedades Infecciosas y Microbiologica Clinica 2011; 29: 502509. doi: 10.1016/j.eimc.2011.03.005.

31. Karstaedt AS, et al. Extrapulmonary tuberculosis amongst adults: experience at Chris Hani Baragwanath Academic Hospital, Johannesburg, South Africa. South African Medical Journal 2013; 104: 22-24. doi: 10.7196/samj.6374.

32. Al-Hajoj S, et al. Exploring the sociodemographic and clinical features of extrapulmonary tuberculosis in Saudi Arabia. PLoS ONE 2015; 10: e0101667. doi: 10.1371/journal.pone.0101667.

33. Ferreiro L, San José E, Valdés L. Tuberculous pleural effusion. Archivos de Bronconeumologica 2014; 50: 435-443. doi: 10.1016/j.arbres.2013.07.006.

34. Lusiba JK, et al. Evaluation of Cepheid's Xpert MTB/ Rif test on pleural fluid in the diagnosis of pleural tuberculosis in a high prevalence HIV/TB setting. PLOS ONE 2014; 9: e102702. doi: 10.1371/journal.pone.0102702.
35. Meldau R, et al. Comparison of same day diagnostic tools including gene xpert and unstimulated IFN-y for the evaluation of pleural tuberculosis: a prospective cohort study. BMC Pulmonary Medicine 2014; 14: 58. doi: 10.1186/1471-2466-14-58.

36. Ray S, et al. Diagnosis and management of miliary tuberculosis: current state and future perspectives. Therapeutics and Clinical Risk Management 2013; 9: 9-26. doi: 10.2147/TCRM.S29179.

37. Sharma SK, et al. Miliary tuberculosis: new insights into an old disease. Lancet Infectious Diseases 2005; 5: 415 430. doi: 10.1016/S1473-3099(05)70163-8.

38. Lewis DK, et al. Clinical indicators of mycobacteraemia in adults admitted to hospital in Blantyre. Malawi Medical Journal 2003; 15: 56-60.

39. Hadad DJ, et al. Mycobacteraemia among HIV-1-infected patients in São Paulo, Brazil: 1995 to 1998. Epidemiology and Infection 2004; 132: 151-155.

40. Wibaux $\mathbf{C}$, et al. Changes in the incidence and management of spinal tuberculosis in a French university hospital rheumatology department from 1966 to 2010. Joint, Bone, Spine: Revue du Rhumatisme 2013; 80: 516-519. doi: 10.1016/j.jbspin.2012.10.001.

41. Berenguer $\mathbf{J}$, et al. Tuberculous meningitis in patients infected with the human immunodeficiency virus. New England Journal of Medicine 1992; 326: 668-672. doi: 10.1056/NEJM199203053261004.

42. Kourbatova EV, et al. Risk factors for mortality among patients with extrapulmonary tuberculosis at an academic inner-city hospital in the US. European Journal of Epidemiology 2006; 21: 715-721. doi: 10.1007/ s10654-006-9060-7.

43. Bor $\mathbf{J}$, et al. Increases in adult life expectancy in rural South Africa: valuing the scale-up of HIV treatment. Science 2013; 339: 961-965. doi: 10.1126/science.1230413.

44. Dye C, et al. Prospects for tuberculous elimination. Annual Reviews in Public Health 2013; 34: 271-286. doi: 10.1146/annurev-publhealth-031912-114431.

45. Dodd PJ, et al. Predicting the long-term impact of antiretroviral therapy scale-up on population incidence of tuberculosis. PLoS ONE 2013; 8: e75466. doi: 10.1371/journal.pone.0075466. 